\title{
RESISTÊNCIA TÊNSIL DE AGREGADOS E COMPRESSIBILIDADE DE UM SOLO CONSTRUÍDO COM PLANTAS DE COBERTURA EM ÁREA DE MINERAÇÃO DE CARVÃO EM CANDIOTA, RS $^{(1)}$
}

\author{
Diony Alves Reis ${ }^{(2)}$, Cláudia Liane Rodrigues de Lima ${ }^{(3)}$ \& Eloy Antonio Pauletto ${ }^{(3)}$
}

\begin{abstract}
RESUMO
A recuperação de áreas degradadas após a mineração de carvão é fundamental para a sustentabilidade do meio ambiente. Este trabalho objetivou avaliar a qualidade estrutural de um solo construído sob diferentes plantas de cobertura, utilizando como referência um solo construído sem plantas de cobertura e um solo natural sob vegetação nativa à frente da mineração. Concluiu-se que as plantas de cobertura, principalmente a Tanzânia (Panicum maximum), contribuíram para melhoria da qualidade estrutural do solo construído, reduzindo a densidade do solo, promovendo a macroporosidade, a porosidade total, o teor de carbono orgânico total e do diâmetro médio dos agregados estáveis. Mais estudos precisam ser realizados enfocando a agregação, resistência tênsil de agregados e friabilidade, considerando que o método de construção interfere na qualidade estrutural desses solos.
\end{abstract}

Termos de indexação: densidade do solo, porosidade do solo, qualidade estrutural, carbono orgânico.

SUMMARY: TENSILE STRENGTH OF AGGREGATES AND COMPRESSIBILITY

OF A SOIL BUILT UP WITH COVER CROPS IN A COAL MINING AREA IN CANDIOTA, RS, BRAZIL

Land reclamation after coal mining is essential for the sustainability of that environment. This study aimed to evaluate the structural quality of a soil built up under different cover crops, taking a soil built up without cover crops and natural soil under native vegetation across

(1) Extraído da dissertação de mestrado do primeiro autor e apresentada ao Programa de Pós-Graduação em Agronomia, da Faculdade de Agronomia "Eliseu Maciel" - FAEM, da Universidade Federal de Pelotas - UFPel. Recebido em 26 de julho de 2013 e aprovado em 6 de janeiro 2014.

(2) Doutorando do Programa de Pós-Graduação em Manejo e Conservação do Solo e da Água, FAEM - UFPel. Campus Universitário, s/n. Caixa Postal 354. CEP 96010-900 Pelotas (RS). Bolsista da CAPES. E-mail: dionyodin@gmail.com

(3) Professor(a) do Departamento de Solos, FAEM - UFPel. E-mail: clrlima@yahoo.com.br, pauletto_sul@yahoo.com.br 
from the mining operation as references. It may be concluded that cover crops, especially Tanzania (Panicum maximum), contributed to improvement of the structural quality of the built-up soil, reducing bulk density and increasing macroporosity, total porosity, total organic carbon content and the average diameter of stable aggregates. More studies are needed with a focus on aggregation, tensile strength of aggregates and friability since the way in which the soils are built up affects their structural quality.

Index terms: Bulk density, soil porosity, structural quality, organic carbon.

\section{INTRODUÇÃO}

O carvão mineral é um importante componente da matriz energética mundial e nacional, porém a mineração a céu aberto proporciona impactos ao meio ambiente, passíveis de recuperação. As causas da degradação do solo durante a lavra do carvão caracterizam-se pela remoção da cobertura vegetal e do solo, resultando em grandes volumes de rejeito (Vega et al., 2004), comumente composto de horizonte do solo anteriormente removidos pela cava e por formações rochosas (argilitos ou siltitos), que são sobrepostas às camadas de carvão. $\mathrm{O}$ acondicionamento do rejeito na cava minerada origina os solos construídos, ou minesoil (Schroeder et al., 2010).

Quando comparados aos solos naturais, os solos construídos geralmente apresentam baixa disponibilidade de nutrientes, reduzida taxa de infiltração, baixa condutividade hidráulica e retenção de água (Sheoran et al., 2010), decréscimo de matéria orgânica (Anderson et al., 2008), baixo pH, reduzida atividade biológica e $\mathrm{C}$ microbiano (Renella et al., 2008; Chodak et al., 2009) e teores elevados de óxidos de Fe (Reuter, 1997).

A recuperação das áreas degradadas pela mineração tem sido realizada pela utilização de plantas de cobertura, a fim de minimizar os efeitos da compactação do solo e da erosão, promover o acúmulo de matéria orgânica, o desenvolvimento da fauna do solo e a ciclagem de nutrientes (Josa et al., 2012). Trabalhos pioneiros como os de Michelutti \& Wiseman (1995), Dudka \& Domy (1997) evidenciaram que os solos construídos apresentam limitações ao desenvolvimento das plantas de cobertura em razão do inadequado processo de reconstituição topográfica. A mistura de materiais com diferentes formas e composições favorece a instabilidade pela reduzida força de coesão entre os constituintes desses solos, expondo-os à erosão hídrica e eólica (Michelutti \& Wiseman, 1995).

A avaliação da qualidade estrutural dos solos agrícolas submetidos a manejos diferenciados tem sido realizada por meio de atributos como densidade, porosidade (Neves et al., 2006), distribuição de tamanho de agregados estáveis em água e diâmetro médio ponderado dos agregados (Salton et al., 2008), resistência tênsil dos agregados (RT) (Imhoff et al., 2002), avaliação das curvas de compressão (Lima et al., 2004) e grau de compactação (Reichert et al., 2009).
Essas variáveis têm sido utilizadas para possibilitar a adoção de práticas de manejo economicamente viáveis e ecologicamente menos degradantes em solos agrícolas; entretanto, é necessário analisar as respostas dessas variáveis aos processos de recuperação de solos construídos em áreas de mineração. Esta pesquisa teve por objetivo avaliar a eficiência do processo de recuperação de áreas degradadas pela implantação de diferentes plantas de cobertura em áreas de mineração de carvão em Candiota, RS, utilizando atributos de agregação e de compressibilidade em solos construídos.

\section{MATERIAL E MÉTODOS}

\section{Área de estudo}

O estudo foi desenvolvido em experimento conduzido na área de mineração de carvão da Companhia Riograndense de Mineração (CRM), no município de Candiota, Rio Grande do Sul, cujas coordenadas geográficas são $31^{\circ} 33^{\prime} 51.8^{\prime \prime}$ de latitude sul e $53^{\circ} 43^{\prime}$ 28.1" de longitude oeste. Segundo a classificação de Köppen, o clima da região é do tipo C $f a$, subtropical úmido, com temperatura e precipitação pluvial média anuais de $17^{\circ} \mathrm{C}$ e de $1.400 \mathrm{~mm}$, respectivamente.

O solo construído da área experimental caracterizase pela mistura de horizontes com o predomínio do horizonte $\mathrm{B}$, de textura argilosa, com $311,8 \mathrm{~g} \mathrm{~kg}^{-1} \mathrm{de}$ areia; $216,9 \mathrm{~g} \mathrm{~kg}^{-1}$ de silte e $471,3 \mathrm{~g} \mathrm{~kg}^{-1}$ de argila. O solo natural é classificado como Argissolo Vermelho eutrófico típico (Nunes, 2002) de textura franca, contendo 477,8 $\mathrm{g} \mathrm{kg}^{-1}$ de areia; 271,8 $\mathrm{g} \mathrm{kg}^{-1}$ de silte; $\mathrm{e}$ $250,4 \mathrm{~g} \mathrm{~kg}^{-1}$ de argila, até o horizonte B (Embrapa, 1997).

O delineamento experimental foi de casualização em blocos com quatro repetições, em parcelas de $20 \mathrm{~m}^{2}$ $(4 \times 5 \mathrm{~m})$, sendo os tratamentos: T1: solo construído sob Capim Vaquero [Cynodon dactilon (L) Pers]; T2: solo construído sob Brizanta [Urochloa brizantha (Hochst.) Stapf]; T3: solo construído sob Tanzânia (Panicum maximum Jac); e T4: solo construído sob Humidícola [Urochloa humidicola (Rendle) Scheick]. Como referências, foram utilizadas amostras de um solo construído sem plantas de cobertura (T8) e um solo natural sob vegetação nativa à frente da mineração (T9), ou seja, áreas que serão futuramente mineradas. 


\section{Amostragem do solo}

A amostragem do solo foi realizada em Junho de 2010, nas camadas de 0,00-0,05 e 0,10-0,15 m. A escolha dessas camadas foi determinada em razão da suscetibilidade às alterações físicas decorrentes do sistema radicular das Poáceas, utilizadas como plantas de cobertura.

Foram coletadas oito amostras de solo com estrutura não preservada por tratamento, totalizando 48 amostras (uma amostra $\times$ duas camadas de solo $\times$ quatro blocos $\times$ seis tratamentos), que, em laboratório, foram destorroadas manualmente em seus pontos de fraqueza e secas ao ar, para determinação da distribuição das classes dos agregados estáveis em água; dos macro e microagregados; do diâmetro médio ponderado dos agregados estáveis (DMP); da resistência tênsil de agregados (RT); da friabilidade (F) e do carbono orgânico total (COT).

Para avaliação da RT, foram utilizados 1440 agregados (30 agregados $\times$ duas camadas de solo $\times$ quatro blocos $\times$ seis tratamentos), e, para determinação do COT, selecionaram-se 240 agregados adicionais (cinco agregados $\times$ duas camadas de solo $\times$ quatro blocos $\mathrm{x}$ seis tratamentos).

Amostras de solo com estrutura preservada foram coletadas por meio de anéis volumétricos de $4,8 \mathrm{~cm}$ de diâmetro e $3,0 \mathrm{~cm}$ de altura, totalizando 96 anéis (dois anéis por tratamento $\times$ seis tratamentos por bloco $\times$ quatro blocos $\times$ duas camadas de solo), que, em laboratório, foram saturadas por capilaridade durante $48 \mathrm{~h}$, posteriormente equilibradas em mesa de tensão e, em seguida, secos em estufa por $24 \mathrm{~h}$ para determinação da macroporosidade (Ma); da microporosidade (Mi); da porosidade total (Pt); da densidade do solo (Ds); da pressão de preconsolidação ( $\sigma p)$, do índice de compressão (IC) e do grau de compactação (GC).

\section{Análises físicas do solo}

A densidade do solo foi determinada pelo método do anel volumétrico (Embrapa, 1997), bem como a macroporosidade $(\mathrm{Ma})$ e porosidade total $(\mathrm{Pt})$ pelo método da mesa de tensão, utilizando uma sucção de coluna de água de $0,60 \mathrm{~m}$.

As classes de agregados estáveis em água e o DMP foram estabelecidos conforme Kemper \& Rosenau (1986), modificado por Palmeira et al. (1999), com a utilização do aparelho de oscilação vertical de Yoder (1936). Os macro e microagregados foram quantificados segundo Tisdall \& Oades (1982), considerando o diâmetro de agregados de $0,25 \mathrm{~mm}$ como limite entre essas duas classes de agregados.

Para a definição da RT, utilizou-se um atuador eletrônico linear a uma velocidade constante de $4 \mathrm{~mm} \mathrm{~s}^{-1}$ (MA 933 fabricado pela empresa Marconi LTDA). Antes da aplicação da força, cada agregado foi mensurado quanto à massa e aferido com um paquímetro, obtendo-se o diâmetro médio a partir de sua altura, sua largura e seu comprimento. Após os ensaios, os agregados foram secos em estufa a $105^{\circ} \mathrm{C}$ por $24 \mathrm{~h}$, determinando-se a umidade gravimétrica, conforme Embrapa (1997). Cada agregado foi acomodado na posição mais estável, para a aplicação da carga de $20 \mathrm{kgf}$. $\mathrm{O}$ valor da força aplicada para a ruptura tênsil do agregado foi registrado em um sistema eletrônico de aquisição de dados, sendo a RT calculada conforme Dexter \& Kroesbergen (1985):

$$
\mathrm{RT}=0,576\left(\frac{\mathrm{P}}{\mathrm{D}^{2}}\right)
$$

em que 0,576 representa a constante de proporcionalidade, refletindo a relação entre o estresse compressivo aplicado e o estresse tênsil gerado no interior do agregado; $\mathrm{P}$ é a força aplicada $(\mathrm{N})$ e D é o diâmetro efetivo $(\mathrm{mm})$. O diâmetro efetivo dos agregados (D) foi calculado conforme Watts \& Dexter (1998), sendo:

$$
\mathrm{D}=\mathrm{Dm}\left(\frac{\mathrm{M}}{\mathrm{M}_{0}}\right)^{\frac{1}{3}}
$$

em que Dm o diâmetro médio do agregado ( $\mathrm{mm})$; $\mathrm{M}$, a massa do agregado individual (g) e $\mathrm{M}_{0}$, a massa média dos agregados na população (g).

A friabilidade do solo foi estimada pelo método do coeficiente de variação, proposto por Watts \& Dexter (1998):

$$
\mathrm{F}=\frac{\sigma_{\mathrm{y}}}{\mathrm{Y}} \pm \frac{\sigma_{\mathrm{y}}}{\mathrm{Y} \sqrt{2 \mathrm{n}}}
$$

em que F é a friabilidade do solo; $\sigma_{y}$, o desvio-padrão dos valores da RT; Y, a média dos valores de RT; e n, o número de repetições, sendo o segundo termo o erropadrão do coeficiente de variância. A classificação da $\mathrm{F}$, conforme Imhoff et al. (2002): não friável $(\mathrm{F}<0,10)$, ligeiramente friável $(\mathrm{F}=0,10$ a 0,20$)$, friável $(\mathrm{F}=0,20$ a 0,50$)$, muito friável $(\mathrm{F}=0,50$ a 0,80$)$ e mecanicamente instável $(\mathrm{F}>0,80)$.

Após submetidos ao teste de tensão indireta e manualmente moídos em gral de ágata, os agregados foram individualmente acondicionados em ependorf de $2 \mathrm{~mL}$ para determinação do COT via analisador elementar, Perkin Elmer pelo método de combustão seca.

Em laboratório, as amostras de solo com estrutura preservada foram saturadas com água por capilaridade, durante $48 \mathrm{~h}$, sendo posteriormente submetidas ao potencial $(\psi)$ de $-10 \mathrm{kPa}$ e equilibradas em câmaras pressão de Richards (Klute, 1986). Após o equilíbrio, cada amostra foi pesada e submetida ao ensaio de compressão uniaxial, em consolidômetro automático Modelo CNTA-IHM/BR (Masquetto Automação Agrícola), sendo aplicadas as pressões sucessivas e estáticas de 25, 50,100,200, 400, 800 e $1.600 \mathrm{kPa}$ (Silva et al., 2007). Obtiveram-se as curvas de compressão do solo relacionando o logaritmo da pressão aplicada (eixo das ordenadas) versus a densidade do solo (eixo das abscissas) para avaliação 
da pressão de preconsolidação (Dias Júnior \& Pierce, 1995) e do índice de compressão, conforme Casagrande (1936):

$$
\mathrm{IC}=\left(\frac{\mathrm{Ds}}{\log \sigma \mathrm{p}}\right)
$$

em que Ds é a densidade do solo; e logøp, o logaritmo da pressão de preconsolidação.

Para eliminar o efeito da compactação inicial do solo, os valores foram normalizados, dividindo-se a densidade ao final da aplicação de cada carga (Ds) pela densidade inicial do solo (Dsi).

O grau de compactação do solo foi calculado pela relação:

$$
\mathrm{GC}=\left(\frac{\mathrm{Ds}}{\text { Dsref }}\right) \times 100
$$

em que Ds é a densidade do solo; e Dsref, a densidade do solo após a aplicação de $200 \mathrm{kPa}$, (GC 200), de 1.600 $\mathrm{kPa},(\mathrm{GC1600})$ e da pressão de preconsolidação (GCøp), conforme Reichert et al. (2009).

\section{Procedimentos estatísticos}

Verificou-se a normalidade dos dados por meio do teste de Shapiro-Wilk (W) para variáveis com número de observações inferior a 200 (Razalli \& Wah, 2011). Os valores discrepantes (outliers) foram identificados pela utilização das medidas do limite inferior (LI) e do limite superior (LS), considerando o primeiro quartil (Q1), o terceiro quartil (Q3) e 1,5 amplitude interquartílica (Reichardt \& Timm, 2004). Os tratamentos experimentais foram submetidos à análise de variância (Anova), e as médias comparadas pelo teste de Duncan $(\mathrm{p}<0,05)$. Porém, considerando o fato do tratamento T9 não fazer parte do delineamento experimental, esse não foi inserido no procedimento estatístico Anova, tampouco na comparação de médias. Por meio do coeficiente de correlação de Pearson (r), a $\mathrm{p}<0,05$, verificaram-se as relações entre algumas variáveis, utilizando-se o software SAS (SAS, 1999).

\section{RESULTADOS E DISCUSSÃO}

Neste estudo, os solos construídos sob plantas de cobertura apresentaram valores de densidade (Ds) próximos aos do solo natural. Para o solo construído sem planta de cobertura (T8), a Ds nas camadas superficial e subsuperficial foi 30 e $36 \%$ maior, respectivamente, que a do solo natural (T9) e esse, por sua vez, apresentou o menor valor de Ds, quando comparado aos demais tratamentos (Quadro 1). Maiti \& Ghose (2005) consideraram o valor de $1,91 \mathrm{Mg} \mathrm{m}^{-3}$ como limitante ao desenvolvimento de plantas de cobertura em solos construídos. Lima et al. (2012) apontaram valores de densidade de um solo construído variando de 1,36 a 1,46 $\mathrm{Mg} \mathrm{m}^{3}$, sendo o maior valor encontrado no solo construído sem plantas de cobertura, corroborando com os resultados deste trabalho.

Menores valores de Ds foram observados no solo construído sob Tanzânia (T3), o que pode ser por causa da ação radicular dessa planta de cobertura, caracterizado como cespitoso, ou seja, na forma touceira com elevada densidade radicular em profundidade, diferente das demais Poáceas implantadas, que se desenvolvem de forma decumbente. Não há informações sobre crescimento radicular de plantas de cobertura em áreas de mineração de carvão, principalmente considerando a fisiologia das plantas. Porém, Sarmento et al. (2008) observaram que na camada de 0,10-0,20 m concentravam-se $25 \%$ das raízes de Panicum sp., sob diferentes intensidades de pastejo e doses de adubação nitrogenada.

Verificaram-se, por meio do coeficiente de correlação de Pearson $(r)$, relações inversas entre Ds e porosidade total $(\mathrm{Pt})(\mathrm{r}=-0,68$ e $-0,84, \mathrm{p}<0,01)$ e $\mathrm{Ds}$ e macroporosidade (Ma) $(r=-0,49$ e $-0,46, p<0,05)$ para as duas camadas avaliadas, corroborando com resultados de Newell-Price et al. (2013), que também observaram a mesma relação entre esses parâmetros. Lima et al. (2012) verificaram valores de Ma superiores aos encontrados neste trabalho, o que pode ser em razão de que os autores avaliaram espécies de Poáceas diferentes; entretanto, os autores verificaram que a Pt no solo construído variou de 0,44 a $0,47 \mathrm{~m}^{3} \mathrm{~m}^{-3}$, semelhantes aos encontrados neste trabalho

Houve diferenças entre as médias dos agregados estáveis em água nas diferentes classes e tratamentos, com exceção da classe de diâmetro de 0,250-0,105 mm na camada superficial, que não evidenciou diferença estatística (Quadro 2). De forma geral, na camada de $0,00-0,05 \mathrm{~m}$, nas classes de maior diâmetro $(9,52-4,76$ e de 4,76-2,00 $\mathrm{mm}$ ), o solo construído sem planta de cobertura (T8) apresentou os menores valores percentuais, mas que foram aumentando sua importância com a redução do diâmetro da classe.

$\mathrm{Na}$ camada subsuperficial, evidenciaram-se diferenças estatísticas em todos os tratamentos. O solo construído sob Brizanta (T2) apresentou a menor porcentagem de agregados na classe de 4,76-2,00 $\mathrm{mm}$. No que se refere ao solo construído sem planta de cobertura (T8) nesta camada, a porcentagem de agregados aumentou com a diminuição do diâmetro da classe, assim como na camada superficial (Quadro 2).

Six et al. (2004) apresentaram uma revisão sobre os processos e fatores que envolvem a formação e estabilização dos agregados, ressaltando a importância das forças de atração entre as partículas minerais, entre as partículas minerais e orgânicas, o emaranhado promovido pelas hifas fúngicas, o teor de carbono orgânico total, a presença de microrganismos, o intemperismo, os ciclos de umedecimento e secagem e o manejo do solo. Os autores chamaram atenção para a importância em se definir a hierarquia dos diversos fatores na manutenção dos agregados do solo, 
principalmente no que se refere à presença da matéria orgânica, fator determinante na estabilização dos agregados do solo.
Como os solos construídos possuem características próprias, em razão dos distintos métodos de extração do minério e da construção dos solos, maiores valores

Quadro 1. Densidade (Ds), porosidade total (Pt), macroporosidade (Ma), macroagregados (Macro), microagregados (Micro), diâmetro médio ponderado de agregados estáveis (DMP), resistência tênsil (RT), friabilidade (F), classes de friabilidade e carbono orgânico total (COT) de um solo construído sob diferentes plantas de cobertura, sem plantas de cobertura e um solo natural nas camadas de 0,00-0,05 e 0,10-0,15 m, em área de mineração de carvão

\begin{tabular}{|c|c|c|c|c|c|c|c|c|c|c|}
\hline & Ds & $\mathrm{Pt}$ & Ma & Macro & Micro & DMP & RT & $\mathbf{F}$ & Classe de $\mathrm{F}^{(1)}$ & COT \\
\hline & \multirow[t]{2}{*}{$\mathrm{Mg} \mathrm{m}^{-3}$} & \multicolumn{2}{|c|}{$\mathrm{m}^{3} \mathrm{~m}^{-3}$} & \multicolumn{2}{|c|}{ \% } & $\mathrm{mm}$ & $\mathrm{kPa}$ & & & $\mathrm{g} \mathrm{kg}^{-1}$ \\
\hline \multicolumn{10}{|c|}{$0,00-0,05 \mathrm{~m}$} & \\
\hline $\mathrm{T} 1$ & $1,44 \mathrm{~b}$ & $0,46 \mathrm{a}$ & $0,07 \mathrm{~b}$ & $87,21 \mathrm{a}$ & $12,79 \mathrm{~b}$ & $2,97 \mathrm{a}$ & $70,32 \mathrm{~b}$ & 0,63 & Muito friável & $1,03 \mathrm{ab}$ \\
\hline $\mathrm{T} 2$ & $1,37 \mathrm{c}$ & $0,43 a b$ & $0,05 \mathrm{~b}$ & $81,15 \mathrm{ab}$ & $18,49 \mathrm{a}$ & $2,28 \mathrm{ab}$ & $75,52 \mathrm{ab}$ & 0,67 & Muito friável & $1,18 \mathrm{a}$ \\
\hline T3 & $1,36 \mathrm{c}$ & $0,47 \mathrm{a}$ & $0,09 \mathrm{a}$ & $84,41 \mathrm{ab}$ & $15,59 \mathrm{ab}$ & $2,62 \mathrm{ab}$ & $88,81 \mathrm{a}$ & 0,51 & Friável & $0,89 \mathrm{~b}$ \\
\hline $\mathrm{T} 4$ & $1,44 \mathrm{~b}$ & $0,41 \mathrm{ab}$ & $0,09 \mathrm{a}$ & 87,69 a & $12,31 \mathrm{~b}$ & $3,18 \mathrm{a}$ & $85,80 \mathrm{a}$ & 0,65 & Muito friável & $0,97 \mathrm{~b}$ \\
\hline $\mathrm{T} 8$ & $1,56 \mathrm{a}$ & $0,38 \mathrm{~b}$ & $0,05 \mathrm{~b}$ & $77,57 \mathrm{~b}$ & $22,43 \mathrm{a}$ & $1,62 \mathrm{~b}$ & $69,67 \mathrm{~b}$ & 0,74 & Muito friável & $0,93 \mathrm{~b}$ \\
\hline T9 & 1,20 & 0,45 & 0,12 & 89,15 & 10,85 & 3,24 & 55,98 & 0,56 & Muito friável & 2,04 \\
\hline \multicolumn{11}{|c|}{$0,10-0,15 \mathrm{~m}$} \\
\hline $\mathrm{T} 1$ & $1,47 \mathrm{~b}$ & $0,47 \mathrm{~b}$ & $0,06 \mathrm{a}$ & $80,15 a b$ & $19,85 \mathrm{ab}$ & $3,59 \mathrm{a}$ & $88,35 \mathrm{bc}$ & 0,70 & Muito friável & $0,80 \mathrm{a}$ \\
\hline $\mathrm{T} 2$ & $1,44 \mathrm{~b}$ & $0,47 \mathrm{~b}$ & $0,05 a b$ & $85,25 \mathrm{a}$ & $14,75 \mathrm{~b}$ & $2,93 a b$ & $76,90 \mathrm{c}$ & 0,77 & Muito friável & $0,67 \mathrm{bc}$ \\
\hline T3 & $1,32 \mathrm{c}$ & $0,53 \mathrm{a}$ & $0,06 \mathrm{a}$ & 84,16 a & $15,84 \mathrm{~b}$ & $3,32 \mathrm{a}$ & $125,92 \mathrm{a}$ & 0,53 & Friável & $0,65 \mathrm{c}$ \\
\hline $\mathrm{T} 4$ & $1,49 \mathrm{~b}$ & $0,45 \mathrm{bc}$ & $0,03 \mathrm{~b}$ & $86,92 \mathrm{a}$ & $13,08 \mathrm{~b}$ & $2,76 \mathrm{ab}$ & $86,09 \mathrm{bc}$ & 0,63 & Muito friável & $0,78 \mathrm{ab}$ \\
\hline $\mathrm{T} 8$ & $1,64 \mathrm{a}$ & $0,42 \mathrm{c}$ & $0,03 \mathrm{~b}$ & $72,12 \mathrm{~b}$ & $27,88 \mathrm{a}$ & $2,09 \mathrm{~b}$ & $98,43 \mathrm{~b}$ & 0,58 & Muito friável & 0,66 bc \\
\hline T9 & 1,18 & 0,54 & 0,12 & 80,65 & 19,35 & 3,24 & 66,28 & 0,64 & Muito friável & 1,26 \\
\hline
\end{tabular}

T1: solo construído sob Capim Vaquero; T2: solo construído sob Brizanta; T3: solo construído sob Tanzânia; T4: solo construído sob Humidícola; T8: solo construído sem plantas de cobertura; e T9: solo natural sob vegetação nativa. Médias seguidas pela mesma letra minúscula nas colunas, dentro de cada variável e camada de solo, não diferem significativamente pelo teste de Duncan a 5 \%. (1) Segundo Imhoff et al. (2002).

Quadro 2. Agregados estáveis em água nas diferentes classes de diâmetro de um solo construído sob diferentes plantas de cobertura, sem plantas de cobertura e um solo natural nas camadas de 0,00-0,05 e 0,10-0,15 m, em área de mineração de carvão

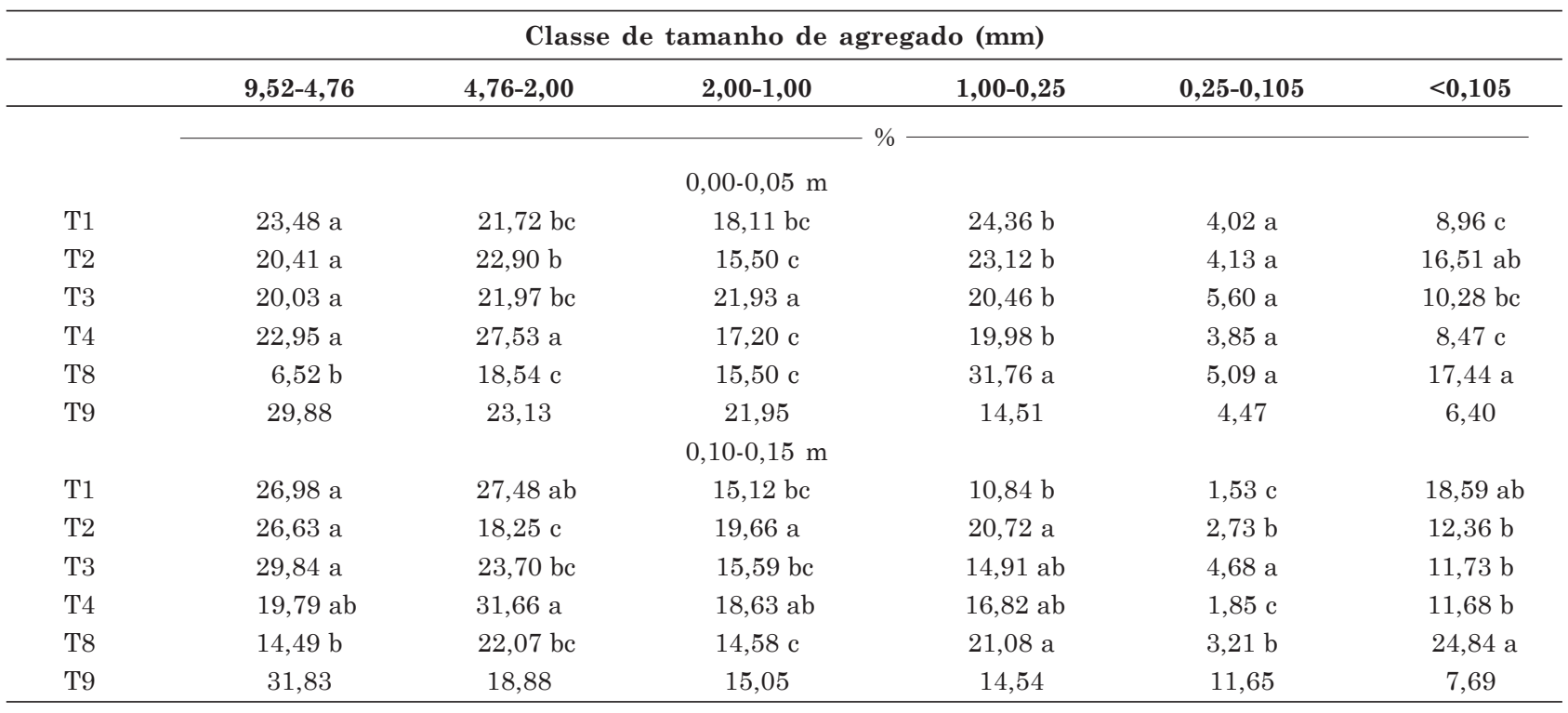

T1: solo construído sob Capim Vaquero; T2: solo construído sob Brizanta; T3: solo construído sob Tanzânia; T4: solo construído sob Humidícola; T8: solo construído sem plantas de cobertura; e T9: solo natural sob vegetação nativa. Médias seguidas pela mesma letra minúscula nas colunas, dentro de cada variável e camada de solo, não diferem significativamente pelo teste de Duncan a $5 \%$. 
percentuais obtidos nas menores classes de diâmetros de agregados podem ser atribuídos ao processo de construção do solo. O trânsito de máquinas de grande porte na construção do solo pode promover adensamento das camadas, reduzindo o volume de poros e o teor de umidade do solo, favorecendo as ligações entre partículas minerais (coesão); entretanto, à medida que o teor de umidade do solo aumenta, as forças de adesão (água - superfície sólida) prevalecem causando o afastamento das partículas minerais originando um sistema disperso, favorável à desagregação do solo, expressando a sensibilidade desses solos à erosão.

O T8 apresentou os menores valores de macroagregados (Macro) e maiores de microagregados (Micro) (Quadro 1). Observou-se que as plantas de cobertura promoveram a agregação e aumentaram o diâmetro dos agregados em razão da exsudação de compostos orgânicos, microfauna presente na rizosfera e por causa da ação mecânica de penetrabilidade do sistema radicular no solo. Todos os tratamentos sob plantas de cobertura apresentaram valores de Macro similares ao solo natural obedecendo à seguinte sequencia: $\mathrm{T} 4>\mathrm{T} 2>\mathrm{T} 3>\mathrm{T} 1>\mathrm{T} 9$, e todos maiores que $\mathrm{o}$ T8. Plantas de crescimento rápido como as Poáceas, que têm sistema radicular diferenciado e que proporcionam melhores índices de cobertura do solo, podem favorecer o processo de recuperação de áreas degradadas, impedindo a desagregação do solo.

Ferreira et al. (2009) verificaram que o aumento no teor de carbono orgânico total (COT) resulta na diminuição da resistência tênsil de agregados (RT), relação inversa e significativa $(p<0,05)$, corroborando com os resultados encontrados neste trabalho. Resultados semelhantes foram obtidos por BlancoCanqui et al. (2005) e Tormena et al. (2008a); porém, contrastaram com os resultados de Imhoff et al. (2002) e Tormena et al. (2008b).

No quadro 1, para as duas camadas avaliadas, todos os tratamentos apresentaram maiores valores de RT quando comparados ao solo natural sob vegetação nativa (T9) e esse, por sua vez, evidenciou maiores valores de COT, embora o coeficiente de correlação entre RT e COT tenha sido negativo e não significativo. Entretanto, como o solo em estudo resultou da mistura de horizontes e formações rochosas, que foi, por meio de maquinário, acondicionada na cava minerada, sobreposta por terra vegetal para posterior implantação das plantas de cobertura, o método de construção do solo influencia de forma inversa a relação entre RT e COT, o que pode ser em razão de as pilhas de solo ficarem por longos períodos de tempo expostas às intempéries, oxidando a matéria orgânica.

Guimarães et al. (2009) afirmaram que a RT, em solos agrícolas, aumenta com o teor de COT. Para Imhoff et al. (2002), a RT é positivamente relacionada com silte, argila, matéria orgânica e óxidos de Fe pouco cristalinos, sendo a friabilidade influenciada pela presença de formas cristalinas de Fe. Chan (1989) relatou que a RT aumenta com a quantidade de argila, dependendo de sua constituição mineralógica.

Neste trabalho, as classes de friabilidade encontradas entre os tratamentos não diferiram, exceto em relação ao T3, o que pode ser atribuído às diferenças fisiológicas dessa planta de cobertura, expressas principalmente pela sua forma de crescimento radicular. Bavoso et al. (2010), avaliando a RT e a friabilidade em diferentes sistemas de preparo de solo agrícola, concluíram que a friabilidade não foi sensível para avaliar diferenças entre os tratamentos, corroborando com informações obtidas neste estudo.

Os valores de COT para todos os tratamentos foram inferiores ao obtido para o solo natural (T9) (Quadro 1). Essa redução nos teores de COT é por causa da remoção da camada fértil do solo. Durante o processo de lavra, a camada superficial (fértil) do solo é retirada, armazenada em pilhas e posteriormente utilizada na reabilitação da área após a lavra, que pode durar vários meses. Nesse tempo, sem aporte de COT, ocorre oxidação da matéria orgânica remanescente no solo. Além das perdas causadas pela oxidação, no preparo do solo minerado para início da reabilitação, esse é misturado com o material do subsolo (estéril e rejeitos), reduzindo ainda mais o teor da matéria orgânica. Em solos de mineração de bauxita na Austrália, Schwenke et al. (2000) verificaram também redução acentuada do teor da matéria orgânica do solo, o que corrobora os resultados encontrados neste estudo.

A revegetação das áreas com Poáceas promoveu aumento no teor de COT em razão da capacidade das plantas de se estabelecerem rapidamente, formando um sistema radicular abundante, facilitando o fluxo de $\mathrm{C}$ fotoassimilado, e da produção de resíduos orgânicos. Esses contribuem para a estruturação, manutenção e proteção do C do solo. Esse desbalanço no ciclo do $\mathrm{C}$ promovido pela mineração evidencia-se reversível com o aumento do tempo de reabilitação e desenvolvimento da vegetação.

O solo construído sem planta de cobertura vegetal (T8) apresentou, nas duas camadas avaliadas, os maiores valores de Ds e o menor índice de vazios (IV), o que pode ser em razão das pressões exercidas sobre o solo (Figura 1), quando no processo de construção desse. Quanto maior o estado de compactação, menor será a deformação do solo após a aplicação de uma pressão, por estar próximo de sua densidade máxima, apresentando menor susceptibilidade à compactação (Suzuki et al., 2008).

Pressões exercidas sobre o solo podem ou não favorecer a compactação, dependendo da capacidade de suporte de carga, expressa pela pressão de preconsolidação, que é indicativo do histórico de pressões exercidas sobre o solo; assim sendo, as curvas elaboradas neste trabalho corroboram com Lima et al. (2012). A pressão de preconsolidação ( $\sigma p)$ e o índice de compressão (IC) não diferiram estatisticamente 
entre os tratamentos nas duas camadas avaliadas (Quadro 3).

O grau de compactação (GC) em diferentes densidades de referência para as duas camadas avaliadas apresentou diferenças entre os tratamentos e, de maneira geral, o T8 evidenciou os maiores valores quando comparados com os outros tratamentos. O GC200 expresso pelo T8 nas duas camadas avaliadas foi 9,3 e $23,4 \%$, maiores que o observado para o solo natural (T9).

Reichert et al. (2009) afirmaram que a compactação interfere nas importantes propriedades do solo, como o fluxo de água e ar, que por sua vez, influenciam a absorção de água e nutrientes pelas plantas, comprometendo o crescimento do sistema radicular. A relação estabelecida entre Pt e GC neste trabalho corrobora com os coeficiente de correlação de Pearson encontrados por Reichert et al. (2009), sendo $r=-0,68, p<0,01$ e $r=-0,62, p<0,01$, para as duas camadas avaliadas. Na figura 2 , verificase os modelos regressivos entre Pt e GC na camada de 0,00-0,05 (a) e 0,10-0,15 m (b).

A densidade na pressão de preconsolidação (Ds $\sigma p$ ) representou a densidade limite que o solo pode

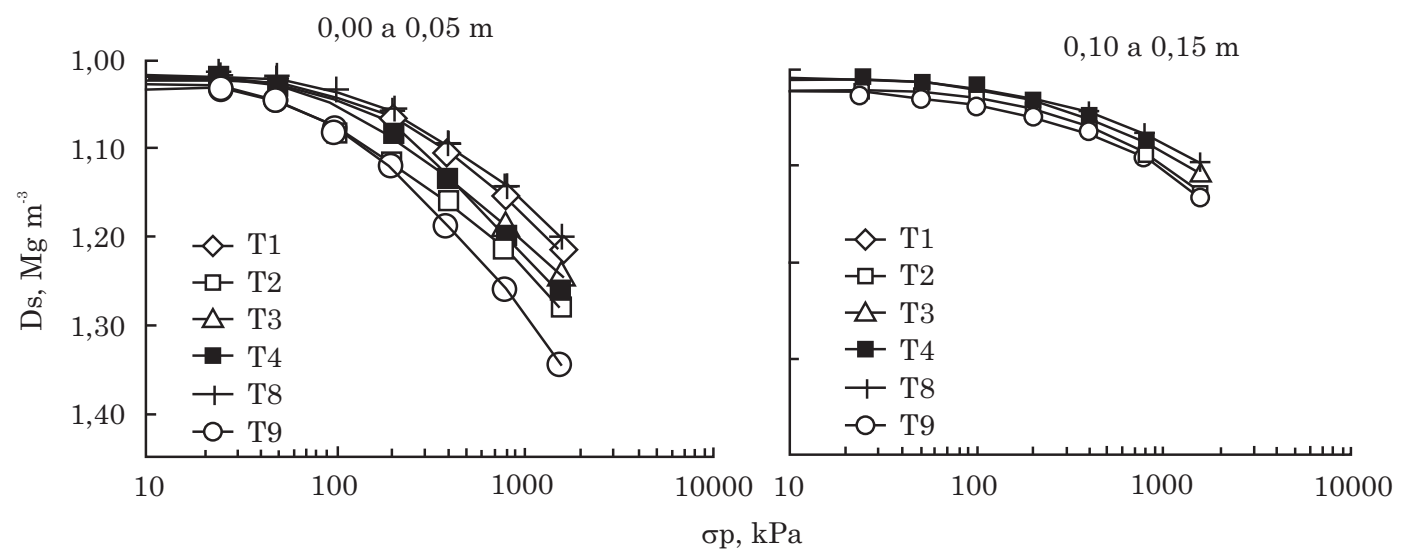

Figura 1. Curvas de compressão normalizadas relacionando a densidade do solo (Ds) em função da pressão aplicada $(\sigma p)$ de um solo construído sob diferentes plantas de cobertura, sem plantas de cobertura e um solo natural nas camadas de 0,00-0,05 e 0,10-0,15 m, em área de mineração de carvão. T1: solo construído sob Capim Vaquero; T2: solo construído sob Brizanta; T3: solo construído sob Tanzânia; T4: solo construído sob Humidícola; T8: solo construído sem plantas de cobertura; e T9: solo natural sob vegetação nativa.

Quadro 3. Pressão de preconsolidação ( $\sigma$ p), índice de compressão (IC), grau de compactação a $200 \mathrm{kPa}$ (GC200), grau de compactação a $1.600 \mathrm{kPa}$ (GC1600), grau de compactação na pressão de preconsolidação $(G C \sigma p)$ e densidade na pressão de preconsolidação (Ds $\sigma p)$ de um solo construído sob diferentes plantas de cobertura, sem plantas de cobertura e um solo natural nas camadas de 0,00-0,05 e 0,10-0,15 m em área de mineração de carvão

\begin{tabular}{|c|c|c|c|c|c|c|}
\hline & $\sigma p$ & IC & GC200 & GC1600 & $\mathrm{GC} \sigma \mathrm{p}$ & Dsop \\
\hline & $\mathrm{kPa}$ & & $\begin{array}{c}\% \\
0,00-0,05 \mathrm{~m}\end{array}$ & $\%$ & $\%$ & $\mathrm{Mg} \mathrm{m}^{-3}$ \\
\hline $\mathrm{T} 1$ & $254,1 \mathrm{a}$ & $0,28 \mathrm{a}$ & $93,9 \mathrm{~b}$ & $82,7 \mathrm{ab}$ & $95,4 \mathrm{ab}$ & $1,51 \mathrm{a}$ \\
\hline $\mathrm{T} 2$ & 203,9 a & $0,31 \mathrm{a}$ & $91,7 \mathrm{~b}$ & $79,9 \mathrm{~b}$ & $97,0 \mathrm{ab}$ & $1,41 \mathrm{a}$ \\
\hline $\mathrm{T} 3$ & $188,6 \mathrm{a}$ & $0,29 \mathrm{a}$ & $91,1 \mathrm{~b}$ & $78,0 \mathrm{~b}$ & $93,9 \mathrm{~b}$ & $1,45 \mathrm{a}$ \\
\hline $\mathrm{T} 4$ & $206,1 \mathrm{a}$ & $0,32 \mathrm{a}$ & $92,8 \mathrm{~b}$ & $79,5 \mathrm{~b}$ & $97,0 \mathrm{ab}$ & $1,48 \mathrm{a}$ \\
\hline $\mathrm{T} 8$ & $238,7 \mathrm{a}$ & $0,27 \mathrm{a}$ & $103,5 \mathrm{a}$ & $91,2 \mathrm{a}$ & $105,1 \mathrm{a}$ & $1,50 \mathrm{a}$ \\
\hline T9 & 176,1 & 0,31 & 93,8 & 76,0 & 92,5 & 1,21 \\
\hline & & & $0,10-0,15 \mathrm{~m}$ & & & \\
\hline $\mathrm{T} 1$ & $286,2 \mathrm{a}$ & $0,18 \mathrm{a}$ & $95,3 b$ & $88,4 \mathrm{~b}$ & $96,4 \mathrm{ab}$ & $1,53 \mathrm{a}$ \\
\hline $\mathrm{T} 2$ & $287,4 \mathrm{a}$ & $0,19 \mathrm{a}$ & $91,0 \mathrm{~b}$ & $84,3 \mathrm{~b}$ & $92,0 \mathrm{~b}$ & $1,57 \mathrm{a}$ \\
\hline $\mathrm{T} 3$ & $286,6 \mathrm{a}$ & $0,16 \mathrm{a}$ & $88,1 \mathrm{~b}$ & $82,3 \mathrm{~b}$ & $89,0 \mathrm{~b}$ & $1,48 \mathrm{a}$ \\
\hline $\mathrm{T} 4$ & $290,6 \mathrm{a}$ & $0,24 \mathrm{a}$ & $94,0 \mathrm{~b}$ & $86,3 \mathrm{~b}$ & $94,9 \mathrm{ab}$ & $1,57 \mathrm{a}$ \\
\hline $\mathrm{T} 8$ & $303,7 \mathrm{a}$ & $0,16 \mathrm{a}$ & $104,5 \mathrm{a}$ & $97,9 \mathrm{a}$ & $103,0 \mathrm{a}$ & $1,60 \mathrm{a}$ \\
\hline T9 & 320,6 & 0,19 & 84,6 & 77,8 & 84,8 & 1,41 \\
\hline
\end{tabular}

T1: solo construído sob Capim Vaquero; T2: solo construído sob Brizanta; T3: solo construído sob Tanzânia; T4: solo construído sob Humidícola; T8: solo construído sem plantas de cobertura; e T9: solo natural sob vegetação nativa. Médias seguidas pela mesma letra minúscula nas colunas, dentro de cada variável e camada de solo, não diferem significativamente pelo teste de Duncan a $5 \%$. 


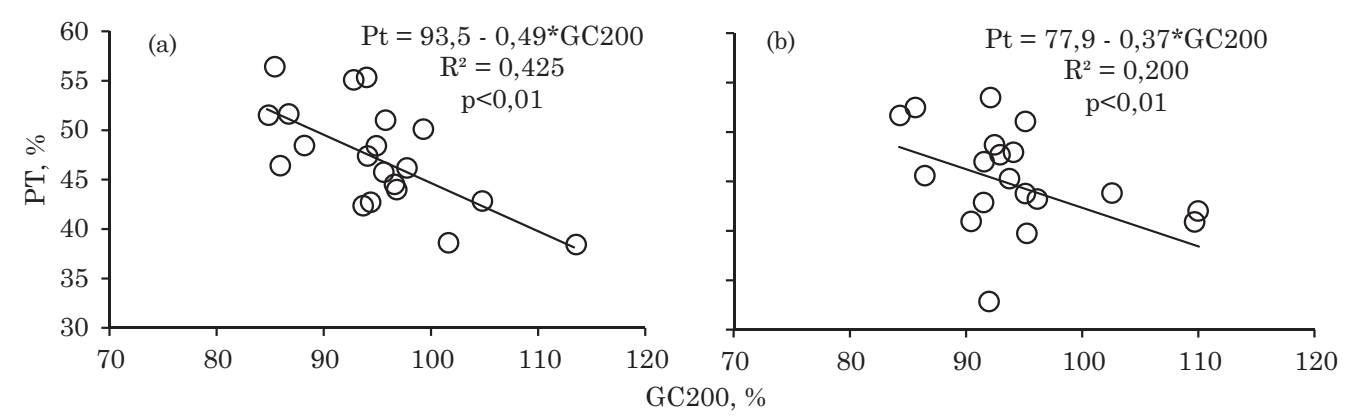

Figura 2. Relação entre porosidade total (Pt) e grau de compactação (GC200) de um solo construído sob diferentes plantas de cobertura nas camadas de 0,00-0,05 m (a) e 0,10-0,15 m (b), em área de mineração de carvão.

apresentar sem que haja incremento de compactação. Relacionando a Ds (Quadro 1) e a Dsøp observou-se que todos os valores de Ds foram menores que a densidade considerada limite, com exceção do T8, que apresentou os valores de Ds maiores que a Dsop, encontrando-se além da capacidade de suporte de carga do solo. Assim sendo, confirmou-se que as plantas de cobertura promoveram melhorias na qualidade estrutural do solo pelo incremento de resíduos orgânicos e pela ação do sistema radicular, favorecendo a Pt e reduzindo a compactação do solo.

O processo de recuperação de áreas degradadas pela utilização de diferentes plantas de cobertura, sobretudo Poáceas, evidenciou-se eficiente na redução da compactação, promovendo melhorias na qualidade estrutural de solos construídos.

\section{CONCLUSÕES}

1. As Poáceas reduzem a densidade do solo, aumentam a macroporosidade, a porosidade total, o diâmetro dos agregados, o teor de carbono orgânico total, reduzindo a compactação dos solos construídos, sendo a planta Panicum maximum Jacq. a mais eficiente no processo de melhoria da qualidade estrutural de um soloconstruído.

2. A resistência tênsil de agregados apresenta relação inversa e não significativa com o teor de carbono orgânico total, e a friabilidade não é sensível às alterações promovidas pelos diferentes tratamentos.

3. O grau de compactação em diferentes densidades de referência e a densidade na pressão de preconsolidação são parâmetros eficientes para inferir sobre a qualidade física de solos construídos.

\section{AGRADECIMENTOS}

ÀUniversidade Federal de Pelotas, pela oportunidade de aprendizado; à Companhia Rio Grandense de Mineração (CRM), pela concessão da área experimental e apoio; à Coordenação de Aperfeiçoamento de Pessoal de Nível Superior (CAPES), pelo suporte financeiro; e a todos os envolvidos direta e indiretamente na realização deste trabalho.

\section{LITERATURA CITADA}

ANDERSON, J.D.; STAHL, P.D. \& INGRAM, L.J. Influence of reclamation management practices on microbial biomass carbon and soil organic carbon accumulation in semiarid mined lands of Wyoming. Appl. Soil Ecol., 40:387-97, 2008.

BAVoso, M.A.; GIAROLA, N.F.B.; TORMENA, C.A. \& PAULETTI, V. Preparo do solo em áreas de produção de grãos, silagem e pastejo: Efeito na resistência tênsil e friabilidade de agregados. R. Bras. Ci. Solo, 34:227-234, 2010 .

BLANCO-CANQUI, H.; LAL, R.; OWENS, L.B.; POST, W.M. \& IZAURRALDE, R.C. Mechanical properties and organic carbon of soil aggregates in the Northern Appalachians. Soil Sci. Soc. Am. J., 69:1472-1481, 2005.

CASAGRANDE, A. The determination of the preconsolidation load and its practical significance. In: INTERNATIONAL CONFERENCE ON SOIL MECHANICS AND FOUNDATION ENGINEERING, D34, 1936, Cambridge. Proceedings... Cambridge, Harvard University, 1936. p.60-64.

CHAN, K.Y. Effect of tillage on aggregate strength and aggregation of Vertisols. Soil Till. Res., 13:163-175, 1989.

CHODAK, M.; NIKINSKA, M. \& PIETRZYKOWSKI, M. Development of microbial properties in a chronosequence of sandy minesoils. Appl. Soil Ecol., 41:259-68, 2009.

DEXTER, A.R. \& KROESBERGEN, B. Methodology for determination of tensile strength of soil aggregates. J. Agric. Eng. Res., 31:139-147, 1985.

DIAS JÚNIOR, M.S. \& PIERCE, F.J. A simple procedure for estimating preconsolidation pressure from soil compression curves. Soil Technol., 8:139-151, 1995. 
DUDKA, S. \& DOMY, C.A. Environmental impacts of metal ore mining and processing: A review. J. Environ. Qual., 26:590-602, 1997.

EMPRESA BRASILEIRA DE PESQUISA AGROPECUÁRIA EMBRAPA. Centro Nacional de Pesquisa de Solos. Manual de métodos de análise de solos. 2.ed. Rio de Janeiro, 1997. p.212.

FERREIRA, A.O.; SÁ, J.C.M.; GIAROLA, N.F.B.; HARMS, M.G. \& BRIEDIS, C. Resistência tênsil de agregados afetada pelo conteúdo de carbono orgânico em um Latossolo Vermelho com textura média e argilosa sob plantio direto. Synerg. Scyent., 4:1-3, 2009.

GUIMARÃES, R.M.L.; TORMENA, C.A.; ALVES, S.J.; FIDALSKI, J. \& BLAINSKI, É. Tensile strength, friability and organic carbon in an Oxisol under a crop-livestock system. Sci. Agric., 66:499-505, 2009.

IMHOFF, S.; PIRES da SILVA, A. \& DEXTER, A. Factors contributing to the tensile strength and friability of Oxisols. Soil Sci. Soc. Am. J., 66:1656-1661, 2002.

JOSA, R.; JORBA, M. \& VALLEJO, V.R. Opencast mine restoration in a Mediterranean semiarid environment: Failure of some common practices. Ecol. Eng., 42:183191, 2012.

KEMPER, W.D. \& ROSENAU, R.C. Aggregate stability and size distribution. In: KLUTE, A., ed. Methods of soil analysis. 2.ed. Madison, American Society of Agronomy/ Soil Science Society of America, 1986. p.425-441.

KLUTE, A. Water retention: Laboratory methods. In: KLUTE, A., ed. Methods of soil analysis: Physical and mineralogical methods. 2.ed. Madison, America Society of Agronomy, 1986. p.635-662.

LIMA, C.L.R.; MIOLA, E.C.C.; TIMM, L.C.; PAULETTO, E.A. \& SILVA, A.P. Soil compressibility and least limiting water range of a constructed soil under cover crops after coal mining in Southern Brazil. Soil Till. Res., 124:190-195, 2012.

LIMA , C.L.R.; SILVA, A.P.; IMHOFF S. \& LEÃO, T.P. Compressibilidade de um solo sob sistemas de pastejo rotacionado intensivo irrigado e não irrigado. R. Bras. Ci. Solo, 28:945-951, 2004.

MAITI, S.K. \& GHOSE, M.K. Ecological restoration of acidic coal-mine overburden dumps - an Indian case study. Land Contam. Reclam., 13:361-369, 2005.

MICHELUTTI, B. \& WISEMAN, M. In: LAL, R. \& STEWART, B.A., eds. Environmental restoration of the industrial city. Berlin, Springer-Verlag, 1995. p.135-141.

NEVES, C.S.V.J.; FELLER, C. \& KOUAKOUA, E. Efeito do manejo do solo e da matéria orgânica solúvel em água quente na estabilidade de agregados de um Latossolo Argiloso. Ci. Rural, 36:1410-1415, 2006.

NEWELL-PRICE, J.P.; WHITTINGHAM, M.J.; CHAMBERS, B.J. \& PEEL, S. Visual soil evaluation in relation to measured soil physical properties in a survey of grassland soil compaction in England and Wales. Soil Till. Res., 127:65-73, 2013.
NUNES, M.C.D. Condições físicas de solos construídos na área de mineração de carvão de Candiota-RS. Pelotas, Universidade Federal de Pelotas, 2002. 130p. (Dissertação de Mestrado)

PALMEIRA, P.R.T.; PAULETTO, E.A.; TEIXEIRA, C.F.A. \& GOMES, A.S., orgs. Pesquisa de Solos (Rio de Janeiro, RJ). Manual de métodos de análise de solos. 2.ed. Rio de Janeiro, 1999. 212p.

RAZALLI, N.M. \& WAH, Y.B. Power comparisons of ShapiroWilk, Kolmogorov-Smirnov, Lilliefors and AndersonDarling tests. J. Stat. Model. An., 2:21-33, 2011.

REICHARDT, K. \& TIMM, L.C. Solo, planta e atmosfera: Conceitos, processos e aplicações. São Paulo, Manole, 2004. p.478.

REICHERT, J.M.; SUZUKI, L.E.A.S.; REINERT, D.J.; HORN, R. \& HAKANSSON, I. Reference bulk density and critical degree-of-compactness for no-till crop production in subtropical highly weathered soils. Soil Till. Res., 102:242$254,2009$.

RENELlA, G.; PIETRAMELlaRA, G.; MENCH, M.; NANNIPIERI, P.; LANDI, L. \& ASCHER, J. Long-term effects of aided phytostabilisation of trace elements on microbial biomass and activity, enzyme activities, and composition of microbial community in the Jales contaminated mine spoils. Environ. Pollut., 152:702-12,2008.

REUTER, R. Sewage sludge as an organic amendment for reclaiming surface mine wastes. 1997. Disponível em: <http://www.hort.agri.umn.edu/h5015/97papers/ reuter.htm>. Acesso em jun. 2013.

SALTON, J.C.; MIELNICZUK, J.; BAYER, C.; BOENI, M.; CONCEIÇÃO, P.C.; FABRÍCIO, A.C.; MACEDO, M.C.M. \& BROCH, D.L. Agregação e estabilidade de agregados do solo em sistemas agropecuários em Mato Grosso do Sul. R. Bras. Ci. Solo, 32:11-21, 2008.

SARMENTO, P.; RODRIGUES, L.R.A.; LUGÃO, S.M.B.; CRUZ, M.C.P.; CAMPOS, F.P.; FERREIRA, M.E. \& OLIVEIRA, R.F. Sistema radicular do Panicum maximum Jacq. cv. IPR-86 Milênio adubado com nitrogênio e submetido à lotação rotacionada. R. Bras. Zootec., 37:27-34, 2008.

SCHROEDER, P.D.; DANIELS, W.L. \& ALLEY, M.M. Chemical and physical properties of reconstructed mineral sand mine soils in Southeastern Virginia. Soil Sci., 175:2-9, 2010.

SCHWENKE, G.D.; MULLIGAN, D.R. \& BELL, L.C. Soil stripping and replacement for the rehabilitation of bauxite-mined land at Weipa. I. Initial changes to soil matter and related parameters. Aust. J. Soil Res., 38:345$369,2000$.

SHEORAN, V.; SHEORAN, A.S. \& POONIA, P. Soil reclamation of abandoned mine land by revegetation: a review. Inter. J. Soil Sed. Water, 3:2-13, 2010.

SILVA, R.B.; MASQUETTO, B.J. \& LANÇAS, K.P. Desenvolvimento e automação de um consolidômetro com interface homem máquina. In: CONGRESSO BRASILEIRO DE CIÊNCIA DO SOLO, 31., Gramado, 2007. Anais... Gramado, Conquistas e Desafios da Ciência do Solo Brasileira, 2007. CD-ROM 
SIX, J.; BOSSUYT, H.; DEGRYZE, S. \& DENEF, K. A history of research on the link between (micro)aggregates, soil biota, and soil organic matter dynamics. Soil Till. Res., 79:7-31, 2004.

STATISTICAL ANALYSIS SYSTEM INSTITUTE - SAS/ STAT. Procedure guide for personal computers; version 9. Cary, 1999.

SUZUKI, L.E.A.S.; REINERT, D.J.; REICHERT, J.M. \& LIMA, C.L.R. Estimativa da susceptibilidade à compactação e do suporte de carga do solo com base em propriedades físicas de solos do Rio Grande do Sul. R. Bras. Ci. Solo, 32:963-973, 2008.

TISDALL, J.M. \& OADES, J.M. Organic matter and water stable aggregates in soil. Eur. J. Soil Sci., 33:141-163, 1982 .
TORMENA, C.A.; ARAÚJO, M.A.; FIDALSKI, J.; IMHOFF, S. \& SILVA, A.P. Quantificação da resistência tênsil e da friabilidade de um Latossolo Vermelho distroférrico sob plantio direto. R. Bras. Ci. Solo, 32:943-952, 2008b.

TORMENA, C.A.; FIDALSKI, J. \& ROSSI JUNIOR, W. Resistência tênsil e friabilidade de um latossolo sob diferentes sistemas de uso. R. Bras. Ci. Solo, 32:33-42, 2008a.

VEGA, F.A.; COVELO, E.F.; ANDRADE, M.L. \& MARCET, P. Relationships between heavy metals content and soil properties in minesoils. Ann. Chim. Acta, 524:141-150, 2004.

WATTS, C.W. \& DEXTER, A.R. Soil friability: Theory, measurement and the effects of management and organic carbon content. Eur. J. Soil Sci., 49:73-84, 1998.

YODER, R.E. A direct method of aggregate analysis of soil and a study of the physical nature of erosion losses. J. Am. Soc. Agric., 28:337-351, 1936. 